\title{
A simplified financial transmission rights auction in the context of the New Zealand electricity grid
}

\author{
Geoffrey Pritchard ${ }^{1} \quad$ Winston L. Sweatman ${ }^{2}$ \\ Ghazaleh Mohammadian $^{3} \quad$ Philip Kilby ${ }^{4}$
}

(Received 15 October 2012; revised 9 October 2013)

\begin{abstract}
This article considers the use of financial transmission rights for managing electricity provision on a power grid. The use of a subgrid of hubs for dealing in these products is explored. Some suitable approaches to this practice are outlined. These identify the feasible size of sub-grid and locate additional constraints.
\end{abstract}

\section{Contents}

http://journal.austms.org.au/ojs/index.php/ANZIAMJ/article/view/6221 gives this article, (c) Austral. Mathematical Soc. 2013. Published October 19, 2013, as part of the Proceedings of the 2012 Mathematics and Statistics in Industry Study Group. ISSN 1446-8735. (Print two pages per sheet of paper.) Copies of this article must not be made otherwise available on the internet; instead link directly to this URL for this article. 
2 The New Zealand electricity network context

3 Physical flow and the spot power market

M87

3.1 Example: the single line . . . . . . . . . . . . . . M89

4 Financial transmission rights

M90

4.1 Example: the single line . . . . . . . . . . . . . . M92

4.2 Financial transmission rights trading hubs . . . . . . . M93

5 Initial investigations

M94

6 The size of the problem

M96

7 Extra constraints on sub-nodal systems

M99

8 Simultaneous transfer experiments

M100

9 Discussion and conclusions

M103

References

M104

\section{Introduction}

In an electricity pool market, all market participants simultaneously trade electric power (at any one point in time) at prices which depend on their locations. Such markets are in current use in power systems in North and South America, the Nordic countries, Australia, and New Zealand. The variation of electricity spot prices with location in these markets has resulted in the development of market instruments to hedge the price differences. A financial transmission right (FTR) is a typical such instrument: a contract with a payoff to the holder which depends on the prices at various locations. The most straightforward variety (known as an obligation FTR) has payoff which is a linear combination of spot prices. Most commonly, this is simply a 
difference of the spot prices at two locations; the FTR is then effectively a swap contract, exchanging power at the first location for an equal amount of power at the second. Also popular are option FTRs, for which the payoff depends nonlinearly on the spot prices; these can represent holding the option (but not the obligation) to exchange power in one location for power in another.

FTRs were first proposed by Hogan [1], and have received plenty of attention under various names: they are called fixed transmission rights in the Pennsylvania-Jersey-Maryland market, transmission congestion contracts in New York, and financial congestion contracts in New England.

The payoffs of FTRs are funded by financial surpluses arising in the spot market. Since these surpluses are of finite size, there are constraints on the quantities of FTRs which are able to simultaneously exist. These constraints are coupled across the various kinds of FTRs; they are collectively referred to as the simultaneous feasibility test. It is this test which makes it possible to award FTRs by an auction mechanism: the auctioneer may award any FTRs they choose (for example to maximize the auction revenue) provided the totality of all existing FTRs satisfies the test.

The problem proposed by Transpower for this MISG arises from a proposed FTR scheme in which the locations (or hubs) relevant to FTRs are only a small subset of all the locations at which physical power is traded. Transpower hoped that this situation would make it possible to formulate the simultaneous feasibility test in a much simpler way than usual.

\section{The New Zealand electricity network context}

Figure 1 shows a potential sub-grid of the New Zealand Electricity network. This grid has seven nodes. Some of these are directly connected by electricity transmission routes; others are only indirectly connected through other nodes. 


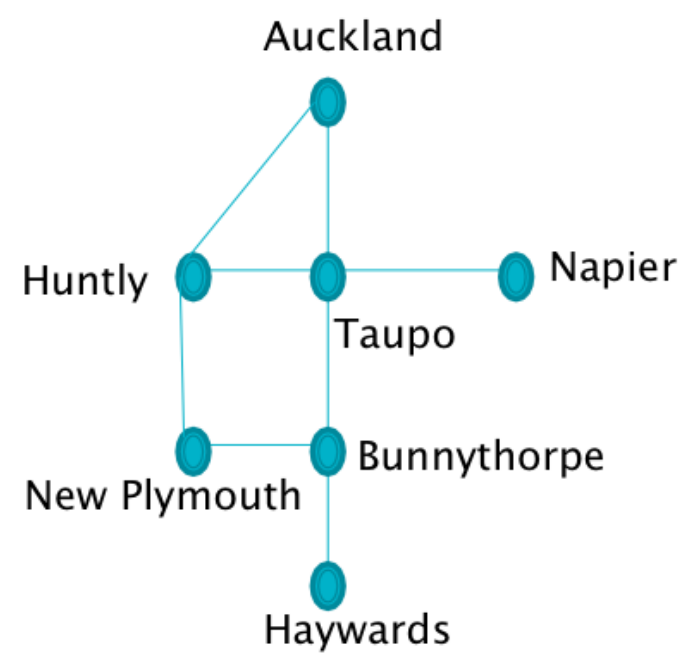

Figure 1: Seven-node subgrid

The Transpower representatives provided the MISG study group with an EXCEL Dispatch and FTR simulator for the seven-node model shown in Figure 1. This program when given a few FTR bids between pairs of nodes calculates the FTRs to be awarded to the bidders.

The usage of power at individual nodes is governed by the physical flow of electricity and the spot market for power. The underlying theory for this is covered in Section 3. The proposed FTR market will be placed onto this existing system. FTR theory is covered in Section 4.

In practice the MISG group began with mixed levels of knowledge of the electricity market. However, progress was made by considering simple cases both with theory and experiments using the Transpower simulator program. The description of the investigation begins in Section 5 . 


\section{Physical flow and the spot power market}

Consider an electric power system comprising a collection $\mathrm{N}$ of nodes (locations) connected by a collection of $L$ transmission lines. Let $\tau_{\mathfrak{n}}(f)$ denote the net power imported via the transmission system to node $n \in N$, when $f=\left(f_{\ell}\right)_{\ell \in L}$ is the vector of line flows. If the lines are lossless, then the $\tau_{\mathrm{n}}$ are linear functions:

$$
\tau_{\mathrm{n}}(\mathrm{f})=\sum_{\ell: v_{1}(\ell)=n} \mathrm{f}_{\ell}-\sum_{\ell: v_{0}(\ell)=n} \mathrm{f}_{\ell}
$$

where $v_{0}(\ell)$ and $v_{1}(\ell)$ are the endpoints of $\ell$, and $f_{\ell}$ is taken to be positive in the direction from $v_{0}(\ell)$ to $v_{1}(\ell)$.

If it is required to model line losses, then nonlinear $\tau_{n}$ are used. A physically realistic choice is a quadratic loss $\rho_{\ell} f_{\ell}^{2}$ on each line:

$$
\tau_{\mathfrak{n}}(f)=\sum_{\ell: v_{1}(\ell)=n}\left(f_{\ell}-\frac{1}{2} \rho_{\ell} f_{\ell}^{2}\right)-\sum_{\ell: v_{0}(\ell)=n}\left(f_{\ell}+\frac{1}{2} \rho_{\ell} f_{\ell}^{2}\right) .
$$

Alternatively, the losses are often modelled as piecewise linear; this is convenient for linear programming formulations.

The capabilities of the transmission system are represented by the requirement $f \in U$. The set $U$ incorporates the maximum capacities of individual lines, loop flow constraints imposed by Kirchoff's laws, and (typically, in practice) other constraints related to contingencies ( $n-1$ security, or some approximation thereof). We assume that $\mathrm{U}$ is a convex compact set with $0 \in \mathrm{U}$.

Given such a system, a pool market for power can be operated as follows. A market operator (MO) is faced with a collection $\mathrm{T}$ of offers to supply or consume electricity. Offer $i \in T$ is for a tranche of quantity $q_{i}$ at a local node $v(i)$; the MO must decide the quantity $x_{i}$ of this to accept. Both $q_{i}$ and $x_{i}$ are taken to be positive in the sense of injecting power to the local 
node, so

$$
x_{i} \in C_{i}:=\left\{\begin{array}{lll}
{\left[0, q_{i}\right],} & \text { if } q_{i} \geqslant 0 & \text { (supply-side offer) } \\
{\left[q_{i}, 0\right],} & \text { if } q_{i} \leqslant 0 & \text { (demand-side bid) }
\end{array}\right.
$$

Offer $i$ also has an associated ask or bid price $p_{i}$; this is taken to be positive when the corresponding cashflow is opposite in direction to the energy flow (which is usually the case). Inelastic demand is handled by setting $p_{i}=$ vOLL, the value of lost load (a high price representing the cost of interrupting supply to a consumer).

The MO's problem is the following.

\section{Problem 1.}

$$
\begin{array}{ll} 
& \min \sum_{i \in T} p_{i} x_{i}, \\
\text { such that } & \tau_{n}(f)+\sum_{i \in T(n)} x_{i}=0 \quad \text { for all } n \in N \\
& x_{i} \in C_{i} \quad \text { for all } i \in T, \\
& f \in U
\end{array}
$$

Here $T(\mathfrak{n})=\{\mathfrak{i}: \boldsymbol{v}(\boldsymbol{i})=\mathfrak{n}\}$. This is an optimization problem in the variables $x=\left(x_{i}\right)_{i \in T}$ and $f$. The problem is convex (that is, it involves minimizing a convex function over a convex set) if the transmission lines are lossless, but may be non-convex if losses are modelled. The most important constraints are those requiring energy balance at node $n$ (that is, $\tau_{n}(f)+\sum_{i \in T(n)} x_{i}=0$ ). The dual variable $\pi_{n}$ associated with such a constraint gives the marginal cost of creating a small power surplus at node $\mathfrak{n}$; this marginal cost becomes the spot price paid or received by market participants at node $n$ whose offers are accepted by the MO.

There is no expectation that the total payments by power consumers should match the total paid to suppliers. Consumers and suppliers are likely to 


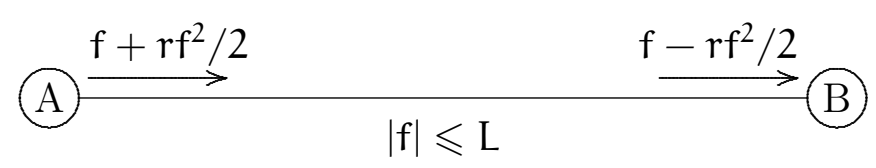

Figure 2: A network consisting of a single lossy line.

be in different locations, so will trade at different prices; furthermore, in a lossy network some of the power supplied will be lost rather than consumed. Philpott and Pritchard [2] show that provided all $\pi_{\mathrm{n}} \geqslant 0$, payments by consumers exceed payments to suppliers by

$$
\sum_{n} \pi_{n} \tau_{n}\left(f^{*}\right)
$$

(where $\pi_{n}$ and $f^{*}$ are taken at optimality in Problem 1 ), and that this quantity is non-negative; that is, no financial deficit can occur.

\subsection{Example: the single line}

Consider, for example, a network consisting of a single line connecting two nodes A and B (Figure 2). The line has quadratic loss coefficient $r$ and capacity L, so that

$$
\tau_{A}(f)=-f-\frac{1}{2} r f^{2}, \quad \tau_{B}(f)=f-\frac{1}{2} r f^{2} \quad \text { and } \quad U=\{f:|f| \leqslant L\} .
$$

(The flow $f$ is taken to be positive in the direction from A to B.) For definiteness, take $\mathrm{L}=100 \mathrm{MW}$ and $\mathrm{r}=0.002 \mathrm{MW}^{-1}$, so that at maximum flow the line must be fed $110 \mathrm{MW}$ at one end and will deliver $90 \mathrm{MW}$ to the other. A supplier at A offers $200 \mathrm{MW}$, asking price $p_{1}=\$ 10 \mathrm{MW}^{-1}$; a similar supplier at $\mathrm{B}$ asks $p_{2}=\$ 20 \mathrm{MW}^{-1}$ for $200 \mathrm{MW}$ there. A consumer at B demands D MW, and is willing to pay a price $p_{3}$ well in excess of $p_{1}$ and $p_{2}$. 
Suppose first that $\mathrm{D}<90$. Then the demand is met entirely by supply from $A$ and use of the transmission line: the flow $f$ is such that $f-r f^{2} / 2=D$, the quantity supplied is $x_{1}=f+\mathrm{rf}^{2} / 2$, and the marginal costs are

$$
\pi_{\mathrm{A}}=\mathrm{p}_{1}, \quad \pi_{\mathrm{B}}=\mathrm{p}_{1} \frac{\mathrm{d} \mathrm{x}_{1}}{\mathrm{dD}}=\mathrm{p}_{1}\left(\frac{1+\mathrm{rf}}{1-\mathrm{rf}}\right)
$$

(since $\frac{d x_{1}}{d D}=\frac{d x_{1}}{d f} / \frac{d D}{d f}$ ). The consumer pays $D \pi_{B}$, the supplier is paid $x_{1} \pi_{A}$, and there is a market surplus equal to

$$
\mathrm{D} \pi_{\mathrm{B}}-\mathrm{x}_{1} \pi_{\mathrm{A}}=\frac{\mathrm{p}_{1} \mathrm{rf} \mathrm{f}^{2}}{1-\mathrm{rf}} .
$$

Note that as D increases from zero to $90 \mathrm{MW}$, the spot price $\pi_{\mathrm{B}}$ increases from $\$ 10 \mathrm{MW}^{-1}$ to $\$ 15 \mathrm{MW}^{-1}$ due to the ever-worsening line losses, while the market surplus increases from zero to $\$ 250$.

Second suppose that $\mathrm{D}>90$. In this case both suppliers must be called upon, with $x_{1}=110, f=100$, and $x_{2}=D-90$. In this regime, the line is said to be congested. The spot prices are $\pi_{A}=p_{1}$ and $\pi_{B}=p_{2}$, and the market surplus is $D \pi_{B}-x_{1} \pi_{A}-x_{2} \pi_{B}=\$ 700$. Note that as $D$ increases through $90 \mathrm{MW}$, both the spot price $\pi_{B}$ and the market surplus change discontinuously.

\section{Financial transmission rights}

A financial transmission right (FTR) is a contract with a payoff to the holder which depends on the spot prices at various locations. An obligation FTR has payoff which is a linear combination of spot prices:

$$
\sum_{n \in N} h_{n} \pi_{n}
$$

for some coefficients $\left(h_{n}\right)$. In the case of a balanced obligation FTR, this is simply a difference of the spot prices at two locations: $\pi_{n_{2}}-\pi_{n_{1}}$; the FTR 
is then effectively a swap contract, exchanging power at the first location for an equal amount of power at the second. An unbalanced obligation FTR is similar except that the quantities swapped are unequal: $h_{2} \pi_{n_{2}}-h_{1} \pi_{n_{1}}$; these occur most often in the context of lossy networks. All the varieties of obligation FTRs sometimes have negative payoff: that is, they may result in a cost to the holder.

An option FTR has payoff:

$$
\left(\sum_{n \in N} h_{n} \pi_{n}\right)_{+}
$$

(where $x_{+}$denotes $\max (x, 0)$ ). In particular, the payoff is always non-negative, which is a simplifying property from a financial perspective.

In the usual market design, the payoffs of financial transmission rights are funded from the surplus that arises in the spot market. This means that it is necessary to limit the quantities of FTRs in existence to ensure that this surplus will be adequate to fund them (revenue adequacy). Where only obligation FTRs are present, the collection of extant FTRs are superposed to produce a single FTR of the form (2); the revenue adequacy requirement is then

$$
\sum_{n \in N} \pi_{n} h_{n} \leqslant \sum_{n} \pi_{n} \tau_{n}\left(f^{*}\right),
$$

where the right-hand side is the spot market surplus discussed in Section 3. A basic result in the theory of FTRs [1,2] states that a sufficient condition for revenue adequacy in this case is the so-called simultaneous feasibility condition: the $h_{n}$ should represent offtakes (or injections) which are feasible for the transmission network. In the notation of the last section, there should exist $f \in U$ with $\tau_{n}(f)=h_{n}$ for each $n$.

Where option FTRs are present, revenue adequacy becomes more onerous to check. One considers all possible ways in which the extant option FTRs may or may not pay off, and checks that in each case, the simultaneous 
feasibility condition remains satisfied when the collection of obligation FTRs is augmented by those option FTRs with non-zero payoff.

Note that the payoffs of FTRs need not correspond directly to flows on particular lines. For example, if an FTR's payoff involves prices at two nodes, and the network admits multiple paths between those nodes, then all paths are relevant to the simultaneous feasibility condition, and to the payoffs achieved.

\subsection{Example: the single line}

Consider a single-line network similar to that in Section 3. An obligation FTR (or superposed collection of several such) with payoff

$$
h_{A} \pi_{A}+h_{B} \pi_{B}
$$

satisfies the simultaneous feasibility condition if and only if

there exists $f:|f| \leqslant L$ and $\left\{h_{A}, h_{B}\right\}=\left\{-f-r f^{2} / 2, f-r f^{2} / 2\right\}$.

That is, $h_{A}$ and $h_{B}$ must represent offtakes (or injections, if negative) which are feasible for the network.

In the case of a lossless line $(r=0)$, it is most natural to consider balanced FTRs. For obligation FTRs only, the payoff would be of form

$$
h_{0}\left(\pi_{B}-\pi_{A}\right)
$$

and the simultaneous feasibility condition reduces to $\left|h_{0}\right| \leqslant L$. Suppose we now add option FTRs with payoffs of form

$$
h_{A B}\left(\pi_{B}-\pi_{A}\right)_{+} \text {and } h_{B A}\left(\pi_{A}-\pi_{B}\right)_{+} .
$$

Exactly one of these options will pay off, so there are two cases to consider; the simultaneous feasibility condition becomes

$$
\left|h_{0}+h_{A B}\right| \leqslant L \text { and }\left|h_{0}+h_{B A}\right| \leqslant L .
$$



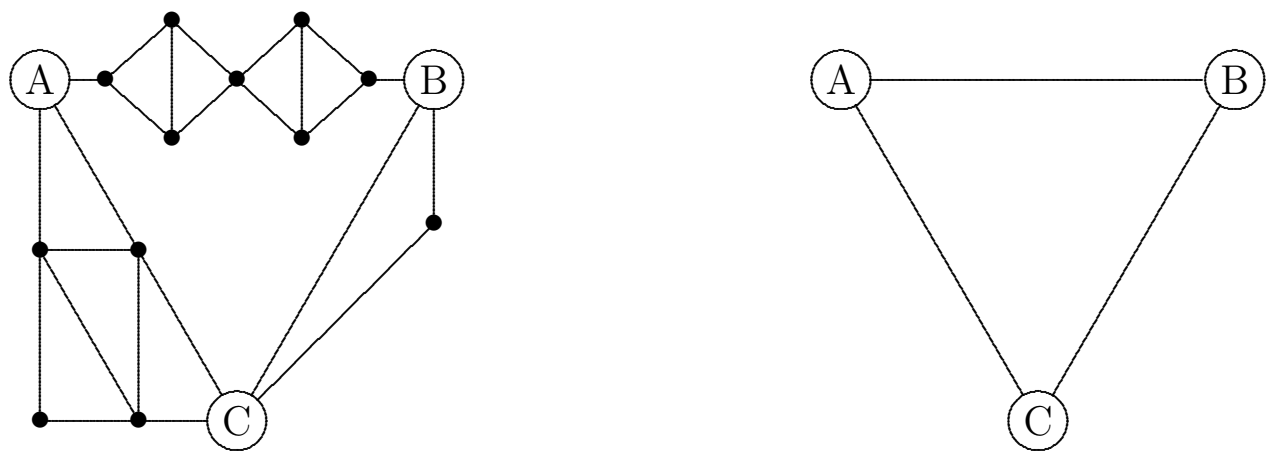

Figure 3: A network with three FTR trading hubs (A,B,C), and its FTR equivalent network.

\subsection{Financial transmission rights trading hubs}

Realistic transmission network models are usually large, with hundreds or thousands of nodes. It may be, though, that only a few of the nodes are involved with FTR payoffs; we refer to these nodes as hubs or trading hubs. The question considered for this MISG is whether the simultaneous feasibility condition can be simplified significantly when the hubs are few in number.

The simultaneous feasibility condition can be simplified significantly in some cases. Consider, for example, the network shown in Figure 3. For the purposes of the simultaneous feasibility test, there will never be any injections or offtakes at nodes which are not hubs, and so the networks connecting A to B, B to C, and $\mathrm{A}$ to $\mathrm{C}$ can each be replaced by a single electrically equivalent line. The simultaneous feasibility test can thus be replaced by a simpler version of itself on a triangular network with three nodes. The remainder of this article considers similar simplifications for other kinds of networks. 


\section{$5 \quad$ Initial investigations}

We took the simplest lossless linear model for our investigations. Consider first a grid with two hubs (Figure 4). The FTR payments relate to the actual electricity price difference between the hubs A and B. However, the redistribution of electric power is constrained by the finite capacity of the power transmission lines between $\mathrm{A}$ and $\mathrm{B}$. Denote by $\mathrm{OP}_{\mathrm{AB}}$ the total quantity of option FTRs accepted from A to B. Use $\mathrm{OB}_{\mathrm{AB}}$ for the total quantity of obligation FTRs from $A$ to $B$. Likewise $\mathrm{OP}_{\mathrm{BA}}$ and $\mathrm{OB}_{\mathrm{BA}}$ are the option and obligation FTRs in the opposite direction from B to A. We write

$$
\begin{aligned}
g_{A B} & =O B_{A B}-O B_{B A}, \\
f_{A B} & =O P_{A B}+O B_{A B}-O B_{B A} \\
& =O P_{A B}+g_{A B},
\end{aligned}
$$

and similarly $g_{B A}$ and $f_{B A}$. (Note $g_{B A}=-g_{A B}$.) Let the grid's transmission capacity from $A$ to $B$ be $Q_{A B}$. That is, $Q_{A B}$ is the maximum value of $x$ such that there is a feasible flow on the grid with injection $x$ at $A$, offtake $x$ at $B$, and no injections or offtakes anywhere else. Then the simultaneous feasibility test (as in Example 4.1) is written

$$
g_{A B} \leqslant Q_{A B}, \quad f_{A B} \leqslant Q_{A B} .
$$

The first of these is redundant as for this two-hub case $g_{A B} \leqslant f_{A B}$ as $O_{A B}$ is always non-negative. There is a similar pair of relations in the opposite direction

$$
g_{B A} \leqslant Q_{B A}, \quad f_{B A} \leqslant Q_{B A} .
$$

One set of relations will be relevant. That will be determined once we know the spot prices at the hubs $\mathrm{A}$ and $\mathrm{B}$ and hence the direction of the flow.

Our aim is to write a similar set of constraints for a grid with more than two hubs, allowing for balanced obligation and option FTRs (in both directions) between all possible pairs of hubs. These constraints need to include interaction terms between the FTRs awarded between different hub-pairs. 


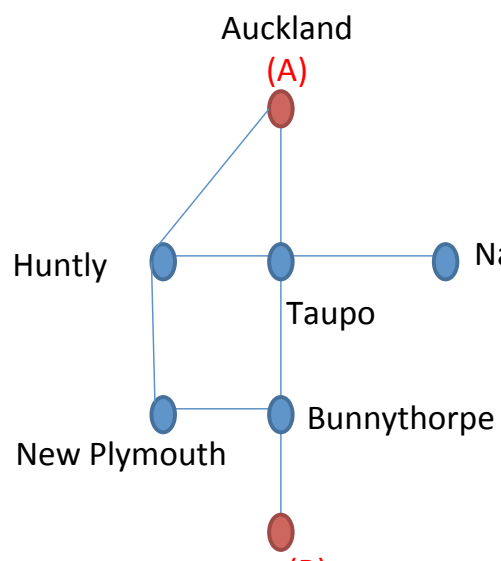

(B)

Haywards

\section{The Grid}

$\mathrm{OB}_{\mathrm{AB}}=$ Obligation FTR from $\mathrm{A}$ to $\mathrm{B}$

$O P_{A B}=$ Option FTR from $A$ to $B$

$\mathrm{p}_{\mathrm{A}}=$ Price at $\mathrm{A}$

\begin{tabular}{|c|c|c|}
\hline & $\begin{array}{c}\text { Northwards } \\
\qquad p_{B}>p_{A}\end{array}$ & $\begin{array}{l}\text { Southwards } \\
\qquad p_{A}>p_{B}\end{array}$ \\
\hline $\mathrm{OB}_{\mathrm{AB}}$ & $\mathrm{p}_{\mathrm{B}}-\mathrm{p}_{\mathrm{A}}$ (positive) & $\mathrm{p}_{\mathrm{B}}-\mathrm{p}_{\mathrm{A}}$ (negative) \\
\hline $\mathrm{OB}_{\mathrm{BA}}$ & $\mathrm{p}_{\mathrm{A}}-\mathrm{p}_{\mathrm{B}}$ (negative) & $\mathrm{p}_{\mathrm{A}}-\mathrm{p}_{\mathrm{B}}$ (positive) \\
\hline$O P_{A B}$ & $p_{B}-p_{A}$ (positive) & zero \\
\hline $\mathrm{OP}_{\mathrm{BA}}$ & zero & $p_{A}-p_{B}$ (positive) \\
\hline
\end{tabular}

Figure 4: Obligation and option FTRs on a simple grid.

Figure 5 shows a three hub system. Flow of electricity from A (Auckland) to $\mathrm{B}$ (Huntly) could be direct or it could be via $\mathrm{C}$ (Taupo). The grid capacity from $A$ to $B\left(Q_{A B}\right)$ allows for all the different routes that the electricity could take. However, there may also be an existing (or required) flow of electricity from A to $\mathrm{C}$. This constrains the quantity of electricity that can flow from A to $\mathrm{B}$ via $\mathrm{C}$. For the three-hub network there are twelve constraint inequalities, one for each direction between each pair of hubs and for each of $f_{A B}$ and $g_{A B}$. For instance, for the case of flow between A and B, we have the relationship

$$
f_{A B}+X_{A C}^{A B} f_{A C}+X_{B C}^{A B} f_{B C} \leqslant Q_{A B}
$$

where $X_{A C}^{A B}$ is the interaction term between the flows on $A B$ and $A C$.

In principle there are inequalities for each combination of directions of potential 


\section{Constraints in a three-hub network}

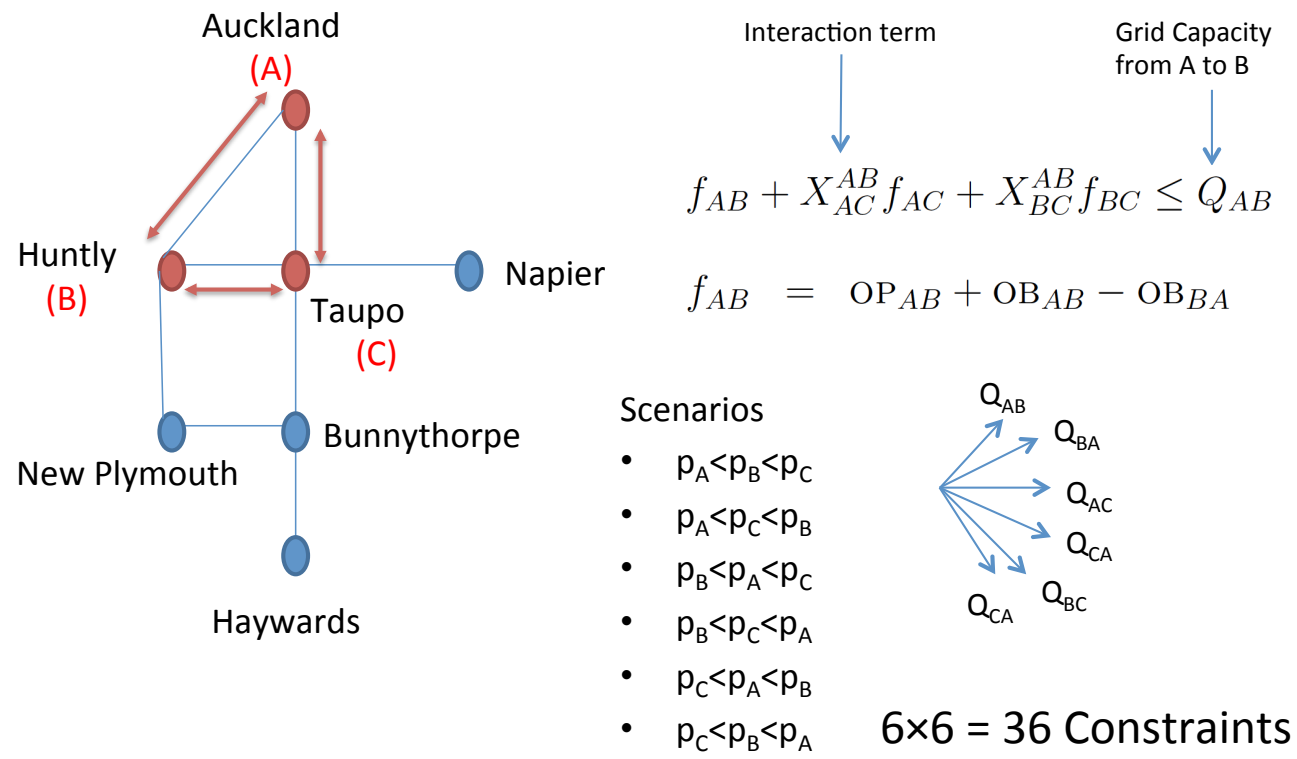

Figure 5: Constraints due to capacity interaction relationships in a three-hub network.

pairwise flow and for both $f_{i j}$ and $g_{i j}$. However, again some of the inequalities are redundant.

\section{The size of the problem}

Consider a larger network of $\mathrm{N}$ hubs labelled $1, \ldots, \mathrm{N}$. The grid capacity between hubs $i$ and $j$ is denoted $Q_{i j}$.

Let $X_{m n}^{i j}$ be the ratio for $i j$ versus $m n$ at the margin (sometimes called 


\section{$i j \times m n)$. We define $X_{i j}^{i j}=1$ for all $i, j$.}

Let all FTR bids be indexed by the set B. The bid price for the FTR $k$ is $p_{k}$ per unit accepted, and the maximum amount required at that price is denoted by $M_{k}$.

Let the indices for bids for product $\mathrm{OP}_{\mathfrak{i j}}$ form the set $\mathrm{I}\left(\mathrm{OP}_{\mathrm{ij}}\right)$. Similarly the bids for $\mathrm{OB}_{\mathfrak{i j}}$ are in the set $\mathrm{B}\left(\mathrm{OB}_{\mathfrak{i j}}\right)$.

The decision variable is $x_{k}$, the quantity of bid $k$ accepted. We also have variables representing the total amount of each financial product accepted: $\mathrm{OP}_{i j}$ representing the total quantity of options between $i$ and $\mathfrak{j}$; and $\mathrm{OB}_{\mathbf{i j}}$ representing the total quantity of obligations between $\boldsymbol{i}$ and $\boldsymbol{j}$.

We use surrogate variables $f_{i j}$ and $g_{i j}$,

$$
\begin{aligned}
g_{i j} & =O B_{i j}-O B_{j i}, \\
f_{i j} & =O P_{i j}+O B_{i j}-O B_{j i} \\
& =O P_{i j}+g_{i j} .
\end{aligned}
$$

Only variables $g_{i j}$ for $i<j$ are defined, and we use the relation $g_{i j}=-g_{j i}$ when $i>j$.

The objective function is then

$$
\operatorname{maximise} \sum_{k \in B} x_{k} p_{k} \text {. }
$$

The first constraints ensure the amount awarded is no more than the amount required

$$
x_{k}<M_{k} \text { for all } k \text {. }
$$


We also define our $f$ and $g$ variables in terms of the decision variables.

$$
\begin{aligned}
g_{i j} & =\sum_{k \in I\left(\mathrm{OB}_{i j}\right)} x_{k}-\sum_{k \in \mathrm{I}\left(\mathrm{OB}_{j i}\right)} x_{k} \text { for all } i<j, \\
f_{i j} & =\sum_{k \in \mathrm{I}\left(\mathrm{OP}_{i j}\right)} x_{k}+g_{i j} \text { for all } i<j, \\
f_{i j} & =\sum_{k \in I\left(\mathrm{OP}_{i j}\right)} x_{k}-g_{j i} \text { for all } i>j .
\end{aligned}
$$

We need to ensure that the total quantities do not exceed the allowed limits. Let $\pi$ be a permutation of the ordering of the hubs. Let $\pi_{i}$ be the ith hub in the permutation. We have the following constraints repeated for each possible permutation $\pi$ :

$$
\begin{aligned}
& \sum_{\mathfrak{m}<n} X_{\pi_{\mathfrak{m}} \pi_{\mathfrak{n}}}^{\pi_{i} \pi_{j}} f_{\pi_{\mathfrak{m}} \pi_{\mathfrak{n}}} \leqslant Q_{\pi_{i} \pi_{j}} \text { for all } i<j, \\
& g_{\pi_{i} \pi_{j}}+\sum_{\substack{m<n \\
\text { except } \\
m=i, n=j}} X_{\pi_{m} \pi_{n}}^{\pi_{j} \pi_{i}} f_{\pi_{m} \pi_{n}} \leqslant Q_{\pi_{j} \pi_{i}} \text { for all } i<j .
\end{aligned}
$$

There are also the non-negativity constraints

$$
f_{i j} \geqslant 0 \text { and } x_{k} \geqslant 0 .
$$

Note that the $g$ variables are not restricted in sign.

The size of the problem can be calculated. The $f$ and $g$ variables and constraints of the form of equations (18) and (19) grow the quickest. These numbers are shown below. The size of the constraint matrix is (number of variables $) \times$ (number of constraints), and is an indication of the size of the linear program. As a general indication, problems of size up to $10^{7}$ are often solved successfully. We see from the table that this corresponds to problems with six or seven hubs. 
Table 1: The size of the linear problem. (For greater than four hubs, the number of constraints and variables $\times$ constraints are given to two significant figures.)

\begin{tabular}{cccc}
\hline $\begin{array}{l}\text { Number of } \\
\text { Hubs }\end{array}$ & $\begin{array}{l}\text { Number of } \\
\mathrm{f}, \mathrm{g} \text { Variables }\end{array}$ & $\begin{array}{c}\text { Number of } \\
\text { Constraints }\end{array}$ & $\begin{array}{c}\text { Variables } \times \\
\text { Constraints }\end{array}$ \\
\hline 2 & 3 & 2 & 6 \\
3 & 9 & 24 & 216 \\
4 & 18 & 216 & 3,888 \\
5 & 30 & $1.9 \times 10^{3}$ & $5.7 \times 10^{4}$ \\
6 & 45 & $1.8 \times 10^{4}$ & $8.1 \times 10^{5}$ \\
7 & 63 & $1.8 \times 10^{5}$ & $1.1 \times 10^{7}$ \\
8 & 84 & $2.0 \times 10^{6}$ & $1.7 \times 10^{8}$ \\
9 & 108 & $2.3 \times 10^{7}$ & $2.5 \times 10^{9}$ \\
10 & 135 & $2.9 \times 10^{8}$ & $4.0 \times 10^{10}$ \\
11 & 165 & $4.0 \times 10^{9}$ & $6.6 \times 10^{11}$ \\
12 & 198 & $5.8 \times 10^{10}$ & $1.1 \times 10^{13}$ \\
\hline
\end{tabular}

\section{Extra constraints on sub-nodal systems}

In addition to the constraints described in the last section, there may be further constraints on a large system. Consider the network in Figure 6. In this network

$$
f_{A C} \leqslant 2, \quad f_{B C} \leqslant 2, \quad X_{A C}^{A B}=0 ;
$$

however,

$$
f_{A C}+f_{B C} \leqslant 3<2+2 .
$$

Expression (22) is a new constraint which does not appear among those considered in Section 6. To see this, note that those constraints all have right-hand sides which are pairwise transfer capacities $Q_{i j}$. In this network, 


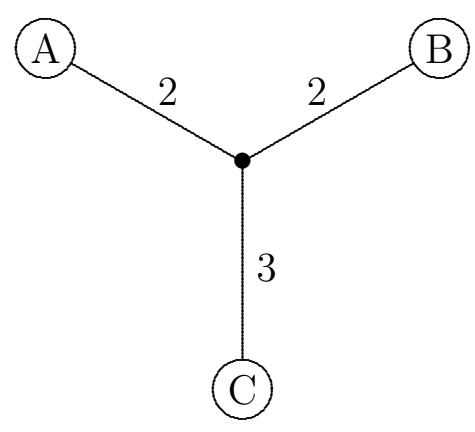

Figure 6: A network which cannot be simplified.

$\mathrm{Q}_{A B}=\mathrm{Q}_{B C}=\mathrm{Q}_{A C}=2$ but in expression (22) we have a constraint with right-hand side 3 .

\section{Simultaneous transfer experiments}

In a lossless grid for which the Problem 1 of Section 3 can be set up and solved, it is possible to explore experimentally the limits of simultaneous feasibility. For example, suppose that we have three hubs A, B and C, and wish to determine the set $K$ of feasible pairs $\left(f_{A B}, f_{A C}\right)$ in the plane. A point $(x, y)$ belongs to $\mathrm{K}$ if and only if there is a flow on the network with injection $x+y$ at $\mathrm{A}$, offtake $\mathrm{x}$ at $\mathrm{B}$, and offtake $\mathrm{y}$ at $\mathrm{C}$; for a particular $(x, y)$ this is tested numerically by solving Problem 1 .

We considered such an example on the seven-node grid shown in Figure 1, for simultaneous transfers Haywards-Auckland and Haywards-Napier. Since Problem 1 (in the lossless case) is a linear programming problem, the feasible set $\mathrm{K}$ is a polygon in the plane. The boundary of this polygon is determined in the following way. Suppose Problem 1 is set up with an infinite supply of zerocost power at Haywards, a consumer bidding $p_{A}$ per unit at Auckland, and 


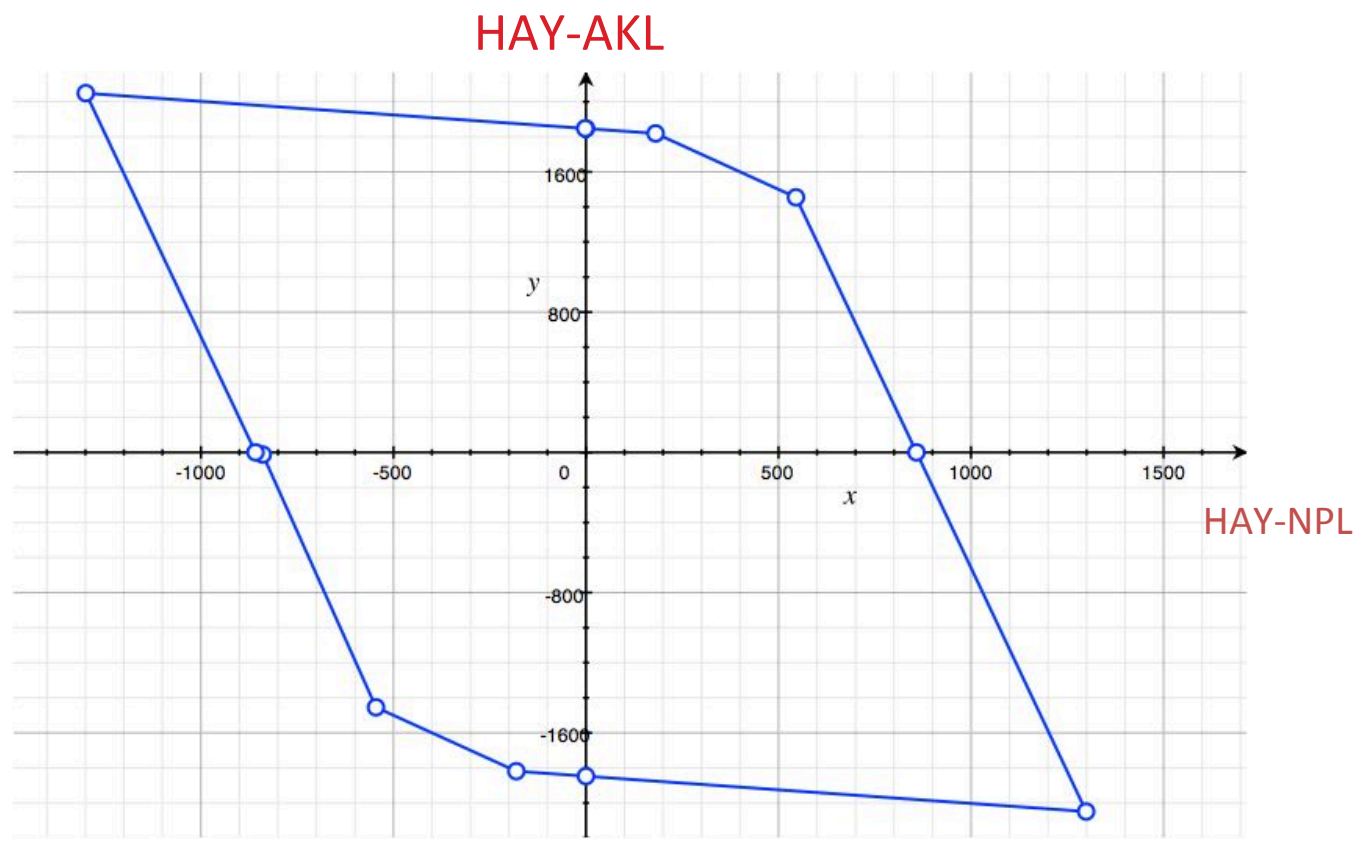

Figure 7: The polygon of constraints for Haywards-Auckland and HaywardsNapier transfers.

another consumer bidding $p_{\mathrm{N}}$ per unit at Napier. The optimal solution will be to supply the two consumers with as much power as possible, subject to the transfer limits of the grid and the relative values of their bids. The resulting flow will be a superposition of transfers $x$ from Haywards to Auckland and y from Haywards to Napier, solving the problem

$$
\max \left\{p_{A} x+p_{N} y:(x, y) \in K\right\} .
$$

Thus, solving the problem determines a point on the boundary of $\mathrm{K}$. By varying the relative values of $p_{A}$ and $p_{N}$, we trace out all the vertices of $K$. This was done experimentally using the Transpower-provided EXCEL Dispatch and FTR simulator (Section 2) to produce Figure 7.

The polygon in Figure 7 is symmetric about the origin $((x, y) \in \mathrm{K} \Longleftrightarrow$ 


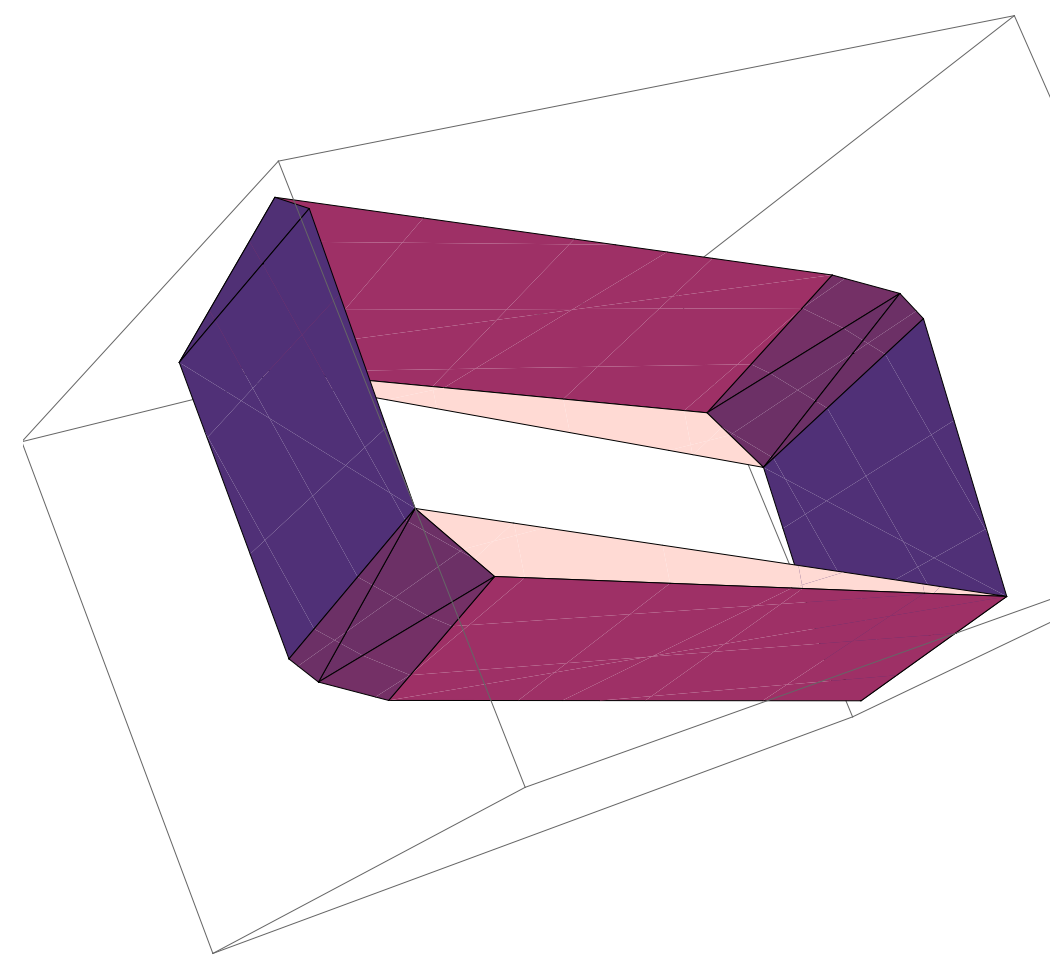

Figure 8: The polyhedron of constraints for Haywards-Auckland, HaywardsNew Plymouth, and Huntly-Auckland transfers.

$(-x,-y) \in K)$. This symmetry is because all the transmission lines in this grid have the same capacity in both directions, so that any feasible flow can be reversed, giving another feasible flow.

Figure 8 shows the result of a similar experiment in which there are three simultaneous transfers being considered: Haywards to Auckland, Haywards to New Plymouth, and Huntly to Auckland. The procedure followed is much the same, resulting in a feasible set which is a polyhedron in three-dimensional space.

Through this mechanism the feasible solution space is constructed for the 
lossless (linear) system, and an optimum is searched for within. Higher dimensional polyhedra would be required when there are larger numbers of links being considered (one for each link). For the lossy system the approach would be complicated by the sides and edges of the polyhedra being curved.

\section{Discussion and conclusions}

The context and work completed on the Transpower problem at the 2012 MISG study group is described. This considered the use of Financial Transmission Rights (FTR) in the context of the New Zealand Electricity Power Grid. In particular, the effectiveness of using a simplified sub-grid of the network for the bidding process is assessed.

The existing operation of the power grid and the mechanism for spot pricing is described. Then the general approach for pricing FTRs is considered. The results of the investigations is described. First a set of constraints is identified that must be satisfied by a simple network with all significant line junctions located at the selected hubs. The system appeared manageable for systems of six or seven hubs. The existence of extra constraints when there are significant line junctions between hubs is then considered. We described an experimental approach for identifying these.

The approach of using a small sub-grid of key hubs to involve in the trading of FTRS appears to be reasonable. Its use will simplify the financial process.

Acknowledgements We are grateful to Transpower and the Industry representatives Conrad Edwards and Vladimir Krichtal for bringing this problem to MISG-2012 and for their valuable input. We also acknowledge and thank the other team members who worked on the problem: Amjad Ali, Alan Brown, Jacobien Carstens, Peter Glover, Zlatko Jovanoski, Sumaira Nasim, Patrick Tobin. The hospitality of our hosts at RMIT University was much appreciated. 


\section{References}

[1] W. W. Hogan, Contract networks for electric power transmission, J. Regulatory Economics 4 (1992), 211-242. doi:10.1007/BF00133621 M85, M91

[2] A. B. Philpott and G. Pritchard, Financial transmission rights in convex pool markets, Oper. Res. Letters, 32 (2004), 109-113. doi:10.1016/j.orl.2003.06.002 M89, M91

\section{Author addresses}

1. Geoffrey Pritchard, Department of Statistics, University of Auckland NeW ZEALAND.

mailto:g.pritchard@auckland.ac.nz

2. Winston L. Sweatman, Centre for Mathematics in Industry, Institute of Natural and Mathematical Sciences, Massey University, Auckland NEW ZEALAND.

mailto:w.sweatman@massey.ac.nz

3. Ghazaleh Mohammadian, School of Computer Science, Engineering and Mathematics, Flinders University, Adelaide Australia. mailto: Ghazaleh.mohammadian@flinders . edu . au

4. Philip Kilby, National ICT Australia and Australian National University, Canberra Australia mailto:Philip.Kilby@nicta.com.au 